\title{
COMMENTARIES
}

\section{Improving Dementia Care Among Family Physicians: From Stigma to Evidence-Informed Knowledge}

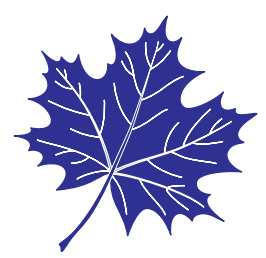

Juanita Bacsu, $\mathrm{PhD}^{1,2}$, Farrah J. Mateen, MD, $\mathrm{PhD}^{3}$, Shanthi Johnson, $\mathrm{PhD}^{4}$, Marc D. Viger, $\mathrm{MD}^{5}$, Paul Hackett, $\mathrm{PhD}^{6}$

${ }^{1}$ Faculty of Kinesiology and Health Studies, University of Regina, Regina, SK and ${ }^{2}$ Saskatchewan Population Health and Evaluation Research Unit (SPHERU), University of Saskatchewan, Saskatoon, SK, Canada; ${ }^{3}$ Department of Neurology, Massachusetts General Hospital, Harvard Medical School, Boston, MA, USA; ${ }^{4}$ School of Public Health, University of Alberta, Edmonton, AB, Canada; ${ }^{5}$ Department of Family Medicine, University of Saskatchewan, Saskatoon, SK, Canada; ${ }^{6}$ Department of Geography, SPHERU, University of Saskatchewan, Saskatoon, SK Canada

https://doi.org/10.5770/cgj.23.426

\section{INTRODUCTION}

Dementia is a rapidly growing public health issue in Canada. Approximately 564,000 Canadians have dementia, and this number is estimated to rise to 937,000 by the year $2031 .^{(1)}$ Age is the greatest risk factor for developing dementia, and the number of Canadians aged 65 years and older is increasing. Recently in 2019, the Government of Canada released a national dementia strategy focused on the need to prevent dementia, advance therapies, find a cure, and overcome stigma to improve the quality of life for people affected by dementia. ${ }^{(2)}$ Stigma refers to an attribute or characteristic which is socially discrediting and may lead to stereotyping, labelling, loss of status, social exclusion, and discriminatory practices. ${ }^{(3)}$ More specifically, the national strategy shares stigmatic experiences from people affected by dementia, ranging from physician reluctance to communicate a diagnosis, to physicians not talking directly to the person with dementia but to the caregiver. ${ }^{(2)}$ Research shows that dementia-related stigma can detrimentally impact interactions with health-care providers, experiences in acute care settings, access to specialist services (e.g., geriatricians and neurologists), and can lead to misdiagnosis, decreased quality of life, depression, and even suicide. ${ }^{(2,4-5)}$

Family physicians are often the primary source of contact in discussing Alzheimer's disease and other forms of dementia, especially in rural and remote communities and provinces with limited access to specialist services. ${ }^{(6)}$ Currently, there are only 300 geriatricians in Canada, with some provinces such as Saskatchewan having access to only one specialist. (7) Ideally, family physicians can provide critical information on cognitive health risk factors, screening and early detection, intervention strategies, prognosis, and supportive care for people with dementia. ${ }^{(8)}$ However, studies show that family physicians are often disinclined to talk about dementia with their patients. ${ }^{(2,3,9)}$

\section{Dementia Care Challenges}

It is well-documented that family physicians report feeling illequipped in providing care for older adults living with dementia. ${ }^{(9-12)}$ Some dementia-care challenges identified by family physicians include time restraints, diagnostic uncertainty, limited resources and diagnostic services, challenges surrounding the complexity of dementia, lack of interdisciplinary teams, gaps in knowledge and skills, inadequate access to specialist services (e.g., geriatricians and neurologists), issues with dementia management regulations (e.g., ability to diagnose and prescribe dementia medications), financial constraints, and a lack of knowledge of support services for people living with dementia. ${ }^{(3,9,13-15)}$ The 2015 Commonwealth Fund Survey of Family Physicians found that only two out of five Canadian doctors feel properly educated and prepared to provide care for older adults with dementia. ${ }^{(12)}$ This lack of education and understanding about dementia can lead to the stigmatization of people affected by dementia which results in barriers to health-care access, diagnosis, and quality of care for people with dementia.

\section{Addressing Dementia-Related Stigma Among Family Physicians}

Dementia-related stigma occurs among students, family members, long-term care workers, nurses, and the general public. ${ }^{(16)}$ Stigma towards people with dementia is also welldocumented among family physicians. ${ }^{(2-5,16-19)}$ Alzheimer Disease International notes that physicians avoid addressing cognitive health concerns because of dementia-related stigma, 
"therapeutic nihilism", and negative views that nothing can be done to help people with dementia. ${ }^{(3)}$ Similarly, existing research highlights that stigmatizing beliefs and inadequate education among family physicians often delay a timely dementia diagnosis and contribute to the widespread underdiagnosis of dementia. ${ }^{(2-5)}$

Dementia-related stigma and negative views among health-care providers need to be addressed as they impede early dementia diagnosis and quality care. ${ }^{(5,13,17)}$ More specifically, some of these negative views include: lack of perceived therapeutic benefits of early dementia diagnosis; giving low priority to dementia symptoms; feelings of helplessness and the view that nothing can be done; concerns of the harmful effects of diagnosis; concerns of further straining the healthcare system; and unwillingness to communicate a dementia diagnosis. ${ }^{(5,10,13,17-18)}$ However, an early diagnosis enables people with dementia to acquire relevant information and support services, plan for the future, engage in cognitive health promotion activities, and access pharmaceutical treatments that may improve their quality of life. ${ }^{(3,5,13)}$

\section{Need to Prioritize Dementia Education and Training}

While most family medicine programs offer some form of education on dementia, few provide an in-depth focus on cognitive health and aging. ${ }^{(3,14)}$ The content of dementia and geriatric education varies by school and ranges from one lecture to an optional, clinical rotation in geriatrics. ${ }^{(14,15)} \mathrm{Put}$ simply, too few family medicine students pursue geriatrics and training opportunities in care of the elderly. ${ }^{(3,15)}$ Moreover, older adults with dementia are diverse, and quality care requires students with clinical training and education that recognizes the unique needs of different geographic (e.g., urban, rural, and remote) and socio-cultural groups.

Given Canada's aging population, there is a critical gap and need for better education and training on dementia in family medicine training programs to improve timely dementia diagnosis, treatment, and quality of care. Specifically, more education on proactive management and risk reduction strategies are vital to addressing stigmatic beliefs, nihilism (e.g., nothing can be done, straining the health-care system, helplessness), and the widespread underdiagnosis of dementia. $(3,13)$ Accordingly, it is important to be educated and abreast of non-pharmacologic interventions to support cognitive health promotion. While there is no known cure for dementia, education on modifiable risk factors is vital as it can delay the onset or slow progression of the disease. ${ }^{(20-21)}$ Recently in 2019, the World Health Organization released guidelines for health-care providers on dementia prevention and risk reduction strategies. ${ }^{(20)}$ Similarly, the Lancet Commission on Dementia Prevention, Intervention, and Care Report identified risk reduction strategies including exercise, education, social engagement, smoking cessation, and management of hearing loss, depression, hypertension, obesity, and diabetes. (21) Consequently, it is essential that dementia education is prioritized in order to reduce stigma and improve cognitive health promotion, timely diagnosis, and quality care for people with dementia.

\section{More Evidence-Informed Knowledge, Support, and Education}

Despite the existing body of literature documenting the stigmatizing attitudes among family physicians, there is a paucity of research on interventions to reduce dementiarelated stigma. There has been substantial research focused on addressing stigma for other diseases such as cancer and mental health. ${ }^{(3,5)}$ Future research should explore effective stigma-reduction strategies and lessons learned from other health fields. Research on stigma prevention and mitigation is necessary to optimize early dementia diagnosis, improve geriatric health services, and facilitate proactive interventions to support cognitive health. More evidence-informed knowledge is required to identify, develop, and evaluate antistigma interventions of dementia among health-care providers, including family physicians.

In working to improve physician education of dementia, an important source of information is the Alzheimer Society of Canada. ${ }^{(1)}$ They offer educational materials on stigmareduction strategies, as well as resources on dementia screening and diagnosis. They also offer the First Link ${ }^{\circledR}$ program which provides an opportunity for health-care providers to refer people to the Alzheimer Society to receive accurate information and support.

In addition to the Alzheimer Society of Canada, there are Continuing Medical Education (CME) programs available to support physician education on dementia and care of the elderly, including Mount Sinai's dementia toolkit for primary care providers, (https://www.mountsinai.on.ca/care/ psych/patient-programs/geriatric-psychiatry/prc-dementiaresources-for-primary-care/dementia-toolkit-for-primarycare), online modules and videos, such as OTN videos, (http:// geriatrics.otn.ca/\#tab1), Ontario's Primary Care Dementia Assessment and Treatment Algorithm Project (https://www. saintlukeskc.org/sites/default/files/2017-12/Medical_Professionas_SLC_Dementia_PC-Data_Assessment_12-17.pdf), and the 5-Weekend Care of the Elderly Certificate Course (https://www.cfp.ca/content/61/3/e135). Although these educational programs exist, improved physician awareness is necessary to promote access and usage of these resources.

In moving forward, there is a strong need for concrete action plans which outline specific steps that can be taken to reduce stigma and improve the quality of health care for people with dementia. Action plans are useful for determining activities, priorities, and timelines, and for demonstrating accountability to support change. While it is outside the reach of this commentary to provide a dementia action plan, an informative example is the Alberta Dementia Strategy and Action Plan. ${ }^{(22)}$ This plan identifies specific actions (e.g., identify and share evidence informed dementia care information, and engage with physicians to review diagnostic needs) and a timeline to improve timely dementia recognition, diagnosis, and clinical management through primary health care. ${ }^{(22)}$ 


\section{Improving Dementia Care Through Interprofessional Collaboration}

In order to reduce dementia-related stigma and improve quality of care, there is a strong need for more interprofessional collaboration among physicians and other health-care professionals such as nurse practitioners, social workers, nutritionists, and occupational therapists. Interprofessional collaboration fosters more robust knowledge and comprehensive understandings of dementia (e.g., biomedical, socio-cultural, and behavioural) by building on different health-care professionals' expertise. ${ }^{(2-4)}$ Although interprofessional collaboration is not new to primary care (e.g., diabetes clinics and osteoporosis clinics), there is a paucity of research focusing on interprofessional clinics to support people with dementia in primary care. ${ }^{(3)}$ For instance, there is little knowledge on the implementation of interprofessional teams to support dementia care in terms of roles and how they best function (e.g., leadership, implementation, and coordination) within primary care settings. Accordingly, future research needs to examine different interprofessional models and collaborative approaches to improve early dementia diagnosis and treatment in primary care.

\section{Conclusion}

The national dementia strategy for Canada emphasizes the need to eliminate dementia-related stigma among primary care physicians as it can create barriers to diagnosis, treatment and care. ${ }^{(2)}$ However, eliminating stigma in the health-care system is neither straightforward nor an easy endeavour. While it is beyond the scope of this commentary to provide a comprehensive action plan to eradicate dementia-related stigma, we emphasize that there is a critical need for action to reduce stigma through more evidence-informed research, education, and interprofessional collaboration. Only with this in mind, can we be confident that quality of care for older adults affected by dementia will be improved.

While our discussion focuses on reducing dementia-related stigma among family physicians in Canada, it is important to note that other countries face similar situations including Australia, ${ }^{(9)}$ United States, ${ }^{(23)}$ Ireland, ${ }^{(24)}$ and France. ${ }^{(25)}$

In moving forward to reduce stigma and improve quality of care, we believe that dementia education and training needs to be prioritized in family medicine programs. Dementia education needs to address the diverse health-care needs of different geographic (e.g., urban, rural, and remote) and socio-cultural groups of people with dementia. We suggest that better education on proactive management and risk reduction training is critical to addressing stigmatic beliefs, nihilism, and the widespread underdiagnosis of dementia. Moreover, there is a growing need for more research on antistigma interventions to improve dementia care and timely diagnosis. Further, we emphasize that more interprofessional collaboration is required to address stigma and the complex nature of dementia in primary care settings. Only through the development of more evidence-informed research, education, and interprofessional collaboration can we begin to address issues of stigma and improve health-care access, diagnosis, and quality of care for people living with dementia.

\section{ACKNOWLEDGEMENTS}

This work was supported by the Alzheimer Society of Canada and the Saskatchewan Centre for Patient-Oriented Research.

\section{CONFLICT OF INTEREST DISCLOSURES}

The authors declare that no conflicts of interest exist.

\section{REFERENCES}

1. Alzheimer Society of Canada. Dementia Numbers in Canada. Toronto, ON: Alzheimer Society of Canada, 2019. Available from, https://alzheimer.ca/en/Home/About-dementia/What-isdementia/Dementia-numbers. Accessed 2019 November 10.

2. Government of Canada. A Dementia Strategy for Canada: Together We Aspire. Ottawa, ON: Government of Canada, 2019. Available from, https://www.canada.ca/en/public-health/ services/publications/diseases-conditions/dementia-strategy. html. Accessed 2019 October 18.

3. Alzheimer Disease International. World Alzheimer Report 2012: Overcoming the Stigma Of Dementia. London, UK: ADI, 2012. Available from: https://www.alz.co.uk/research/WorldAlzheimerReport2012.pdf. Accessed 2019 November 12.

4. Harper L, Dobbs BM, Stites SD, et al. Stigma in dementia: it's time to talk about it. Curr Psychiatr. 2019;18(7):16-23.

5. Werner P, Giveon S. Discriminatory behavior of family physicians toward a person with Alzheimer's disease. Int Psychogeriatr. 2008;20(4):824-39.

6. Bacsu J, Novik N, Johnson S, et al. Recognizing the needs of rural and remote people in a national dementia strategy for Canada. Can J Public Health. 2019;110(6):752-55.

7. Glauser W. Lack of interest in geriatrics among medical trainees a concern as population ages. CMAJ. 2019;191(20):E570-E571.

8. Chertkow H. Diagnosis and treatment of dementia: Introduction. Introducing a series based on the Third Canadian Consensus Conference on the Diagnosis and Treatment of Dementia. CMAJ. 2008;178(3):316-21.

9. Pond CD, Brodaty H, Stocks N, et al. P1-164: The attitudes of Australian general practitioners to breaking the news of dementia: a qualitative and quantitative exploration. Alzheimers Dement. 2011;7(4 Suppl 1):S164.

10. Aminzadeh F, Molnar FJ, Dalziel WB, et al. A review of barriers and enablers to diagnosis and management of persons with dementia in primary care. Can Geriatr J. 2012;15(3):85-94.

11. Pimlott NJ, Persaud M, Drummond N, et al. Family physicians and dementia in Canada: Part 2. Understanding the challenges of dementia care. Can Fam Physician. 2009;55(5):508-09.

12. Canadian Institute for Health Information (CIHI). How Canada compares: results from the Commonwealth Fund 2015 International Health Policy Survey of Primary Care Physicians, 2016. Ottawa, ON: CIHI; 2016. Available from: https://www. cihi.ca/sites/default/files/commonwealth_fund_2015_pdf_ en_0.pdf. Accessed 2019 October 8.

13. Petrazzuoli F, Vinker S, Koskela T, et al. Exploring dementia management attitudes in primary care: a key informant survey to primary care physicians in 25 European countries. Int Psychogeriatr. 2017;29(9):1413-23. 


\section{BACSU: FAMILY PHYSICIANS AND DEMENTIA CARE}

14. Gazewood JD, Vanderhoff B, Ackermann R, et al. Geriatrics in family practice residency education: an unmet challenge. Fam Med. 2003;35(1):30-34.

15. Prorok JC, Stolee P, Cooke M, et al. Evaluation of a dementia education program for family medicine residents. Can Geriatr J. 2015;18(2):57-64.

16. Herrmann L, Welter E, Leverenz J, et al. A systematic review of dementia-related stigma research: can we move the stigma dial? Am J Geriatr Psychiatry. 2018;26(3):316-31.

17. Gove D, Downs M, Vernooij-Dassen M, et al. Stigma and GPs' perceptions of dementia. Aging Ment Health. 2016;20(4): 391-400.

18. Vernooij-Dassen MJ, Moniz-Cook ED, Woods RT, et al. Factors affecting timely recognition and diagnosis of dementia across Europe: from awareness to stigma. Int J Geriatr Psychiatry. 2005; 20(4):377-86.

19. Harrison K, Hunt L, Ritchie C, et al. Dying with dementia: underrecognized and stigmatized. J Am Geriatr Soc. 2019;67(8): $1548-51$.

20. World Health Organization (WHO). Risk Reduction of Cognitive Decline and Dementia: WHO Guidelines, 2019. Geneva: World Health Organization; 2019. Available from: https://apps.who. int/iris/bitstream/handle/10665/312180/9789241550543-eng. pdf. Accessed 2019 November 26.
21. Livingston G, Sommerlad A, Orgeta V, et al. Dementia prevention, intervention, and care. Lancet. 2017;390(10113):2673-734.

22. Government of Alberta. Alberta Health. Alberta Dementia Strategy and Action Plan. Edmonton, AB: Alberta Ministry of Health, 2017. Available from: https://open. alberta.ca/dataset/772005d6-94f8-4a62-a39b-cc91265f3fca/ resource/40959fbb-ca1d-4b44-8864-f05e8d1c6d0f/download/ alberta-dementia-strategy-and-action-plan.pdf. Accessed 2020 March 3.

23. Bradford A, Kunik ME, Schulz P, et al. Missed and delayed diagnosis of dementia in primary care: prevalence and contributing factors. Alzheimer Dis Assoc Disord. 2009;23(4):306-14.

24. Cahill S, Clark M, O'Connell H, et al. The attitudes and practices of general practitioners regarding dementia diagnosis in Ireland. Int J Geriatr Psychiatry. 2008;23(7);663-69.

25. Piver LC, Nubukpo P, Faure A, et al. Describing perceived stigma against Alzheimer's disease in a general population in France: the STIG-MA survey. Int J Geriatr Psychiatry. 2013;28(9):933-38.

Correspondence to: Juanita Bacsu, PhD, Saskatchewan Population Health and Evaluation Research Unit (SPHERU), University of Saskatchewan, Saskatoon, SK, Canada S7N 4N3 E-mail: juanita.bacsu@usask.ca 